We will try to publish authors' responses in the same edition with readers' comments. Time constraints might prevent this in some cases. The problem is compounded in a bimonthly journal where continuity of comment and redress are difficult to achieve. When the redress appears 2 months after the comment, 4 months will have passed since the original article was published. Therefore, we would suggest to our readers that their correspondence about published papers be submitted as soon as possible after the article appears.

\section{Approach to Polypharmacy}

To the Editor: I have come to expect commonsense, evidence-based, and cost-effective advice from the $7 A B F P$. The report of a patient uncontrolled on seven antihypertensive medications lacks all three elements, ${ }^{1}$ and the accompanying editorial ${ }^{2}$ fails to add anything.

JNC VI, ${ }^{3}$ though not released prior to the patient's treatment, reflects evidence-based practice recommendations found in previous reviews. According to JNC $\mathrm{VI}$, angiotensin-converting enzyme (ACE) inhibitors, $\alpha$-blockers, calcium antagonists, and diuretics in low doses are preferred for hypertensive diabetic patients. If the patient is not responding adequately, another drug should be added or substituted, and it is suggested that a diuretic be strongly considered if it was not the firstline drug. The patient described was not taking a diuretic but was taking two ACE inhibitors (lisinopril and captopril) and two calcium channel blockers (isradipine and verapamil). This treatment regimen does not make medical sense.

It took the providers in this practice 38 visits before they discovered the patient was not taking her medicines because they cost too much. JNC V and JNC VI suggest that among the most common causes for inadequate blood pressure control is lack of compliance. Common sense dictates that at some time during those 38 visits, someone could have checked compliance by having the patient bring in her bottles to check on the dates and number of pills. Furthermore, we are not told about the patient's heart rate beyond the initial visit, but adequate doses of metoprolol and verapamil should substantially lower it, adding another simple way to check compliance.

Your editorial could have suggested a more reasonable approach to this patient's problems other than the author's solution of continuing her regimen of three expensive drugs supplied by pharmaceutical companies' indigent programs. I have a patient on such a program, and the paperwork hassles every 3 months from each company have convinced me not to do it again. A 6month supply of reserpine and hydrochlorothiazide at my local pharmacy cost $\$ 30.25$ (less than $\$ 0.15$ per day.). We all ignore reserpine, an efficacious but unjustly maligned drug, as we succumb to the glossy ads and hype of the pharmaceutical companies. Fraser ${ }^{4}$ and Lederle et al $1^{5}$ explain how this attitude came about and why reserpine should be part of everyone's armamentarium.

If reserpine and hydrochlorothiazide were not tolerated or did not control the blood pressure, generic propranolol would cost another $\$ 10$ per month. JNC VI points out the potential problems of $\beta$-blockers in diabetic patients but adds that randomized control studies show that patients with diabetes "experience a similar or greater reduction of CHD and total cardiovascular events compared with persons without diabetes." Even if one adds an ACE inhibitor instead, generic captopril would be another $\$ 1$ per day. Second-generation sulfonylureas are no more advantageous than generic chlorpropamide, so the patient's diabetes could be treated for $\$ 0.10$ per day.

I think family physicians can do better than the example in this case report, and I look to the $\mathcal{F A B F P}$ to show us how. In this instance, I was very disappointed.

Gil L. Solomon, $M D$

West Hills, Calif

\section{References}

1. Lee RD. Polypharmacy: a case report and new protocol for management. J Am Board Fam Pract 1998,11:140-4.

2. Meador CK. Polypharmacy: old bad habits. J Am Board Fam Pract 1998,11:166-7.

3. The sixth report of the Joint National Committee on Prevention, Detection, Evaluation, and Treatment of High Blood Pressure, 1997. Bethesda, Md: National IIigh Blood Pressure Education Program, National Institutes of Ilealth, National Heart, Lung, and Blood Institute, 1997. (NIII publication no. 98-4080.)

4. Fraser IIS. Reserpine: a tragic victim of myths, marketing, and fashionable prescribing. Clin Pharmacol Ther 1996;60: 368-73.

5. Lederle FA, Applegate WB, Grimm RII Jr. Reserpine and the medical marketplace. Arch Intern Med 1993;153:705-6.

The above letter was referred to the author of the article in question, who offers the following reply.

To the Editor: I would like to respond to the comments raised by Dr. Solomon concerning my article, "Polypharmacy: A Case Report and New Protocol for Management."

Dr. Solomon raises three points concerning this case report. First, he feels that the initial management of this patient did not "make medical sense." This issue was adequately addressed in my article. In fact, the point of the report was to "describe a patient in whom polypharmacy led to misdiagnosis, unnecessary testing, and inappropriate treatment." Polypharmacy is common in clinical practice, and the intent of the report was to remind family physicians of the pitfalls 
that can arise when polypharmacy is not addressed appropriately.

Dr. Solomon's second concern is the lack of "evidence-based" advice. Admittedly, the SAIL protocol that I proposed "has not yet been tested in clinical practice"; ${ }^{1}$ however, each element in the algorithm is supported in the literature. In much the same way that Dr. Solomon suggests having a patient bring in her bottles and check on the dates and the number of pills, the SAIL protocol is simply a tool that can be used to apply common sense to the problem of polypharmacy.

Finally, Dr. Solomon contends that there are more cost-effective solutions besides "continuing her regimen of three expensive drugs." One must be careful to distinguish between the term cost-effectiveness and the cost of buying a particular drug. Cost-effectiveness takes into account not only the cost of paying for a drug but alse the drug's effectiveness at preventing such other costs as hospitalizations and other morbidity. The drugs that finally controlled this patient's diabetes and hypertension are taken once daily and are associated with a low incidence of adverse effets; as such, they are associated with a higher compliance rate than many less expensive drugs. Her regimen, therefore, is potentially less expensive in the long run. ${ }^{2}$

I want to address one additional point that Dr. Solomon raises: the "paperwork hassles" inherent in pharmaceutical companies' indigent drug programs. $\mathrm{Pa}$ perwork is, unfortunately, a major part of the practice of medicine, but with indigent drug programs we can get tangible results for our hassles. Procuring free medicatons for my indigent patients is worth every second I spend completing the appropriate forms. I believe that taking the time to help patients obtain their medications should be part of every family physician's job.

\section{R. David Lee, MD}

Stanley, Va

\section{References}

1. Lee RD. Polypharmacy: a case report and new protocol for management. J Am Board Fam Pract 1998,11:140-4.

2. Mooradian AD. Drug therapy of non-insulin dependent diabetes mellitus in the elderly. Drugs 1996;51:931-41. 